\title{
Multi-sensor Doppler radar for machine tool collision detection
}

\author{
T. J. Wächter ${ }^{1}$, U. Siart ${ }^{1}$, T. F. Eibert ${ }^{1}$, and S. Bonerz ${ }^{2}$ \\ ${ }^{1}$ Technische Universität München, Lehrstuhl für Hochfrequenztechnik, Arcisstr. 21, 80333 Munich, Germany \\ ${ }^{2}$ Ott-Jakob Spanntechnik GmbH, Industriestr. 3-7, 87663 Lengenwang, Germany
}

Correspondence to: T. J. Wächter (thomas.waechter@tum.de)

Received: 26 January 2014 - Revised: 11 July 2014 - Accepted: 16 July 2014 - Published: 10 November 2014

\begin{abstract}
Machine damage due to tool collisions is a widespread issue in milling production. These collisions are typically caused by human errors. A solution for this problem is proposed based on a low-complexity $24 \mathrm{GHz}$ continuous wave $(\mathrm{CW})$ radar system. The developed monitoring system is able to detect moving objects by evaluating the Doppler shift. It combines incoherent information from several spatially distributed Doppler sensors and estimates the distance between an object and the sensors. The specially designed compact prototype contains up to five radar sensor modules and amplifiers yet fits into the limited available space. In this first approach we concentrate on the Doppler-based positioning of a single moving target. The recorded signals are preprocessed in order to remove noise and interference from the machinery hall. We conducted and processed system measurements with this prototype. The Doppler frequency estimation and the object position obtained after signal conditioning and processing with the developed algorithm were in good agreement with the reference coordinates provided by the machine's control unit.
\end{abstract}

\section{Introduction}

Collisions within the operating space of machine tools have many different causes. Main causes for collisions during human operation are wrong programming (wrong part, compensation or offsets), wrong machine setup (wrong tool, clamping or raw parts) and inattention or faulty operation by the operator himself.

The so called technological collisions concern crashes between tool and work piece only. The second group, the geometric collisions, involves further elements of the machine like the bearings, holder, spindle, axis or the driving shaft.
In the case of tool collisions, tremendous impact forces cause heavy damage to the work piece, the tool and to other elements of the drive train. The results are expensive damage, costs for production downtimes and high rejection rates. The goal is to guarantee high machine availability to maximize efficiency and output.

Up to now, no system is known which reliably provides information in order to avoid collisions with non-moving components and to actively protect the machine components against physical damage. There are some promising sensor based systems that can decouple the inner main motor spindle from the outer spindle stock, hence protecting the components from mechanical overload to reduce damage to a minimum. Other methods, without sensors, monitor the current flux through the motor and driving chain and activate when a certain threshold is exceeded. An elaboration on available safety systems can be found in Abele et al. (2012). All of these are reactive systems which are activated after a crash. With these, mitigation of damage is possible, but not their prevention.

Optical systems like laser scanners or (multi-)camera based systems are active observers. The disadvantage of these systems is that they are blinded due to cooling fluid, drizzle, dust and dirt from the milling treatment which pollute the lens or the sensor space. So their use is limited to phases where the metal processing is stopped or to other applications with non-cutting manufacture.

In this paper, the implementation of a prototype for a new possible solution is presented based on an autonomous active radar sensor safety system which operates in the 24 to 24.25 GHz ISM band. The utilized sensors are commercially available low-cost modules for short-range radar (SRR) applications, containing all front-end parts of a $\mathrm{CW}$ radar, including complex down-conversion. Figure 1 shows the prototype concept, here equipped with four modules in a 


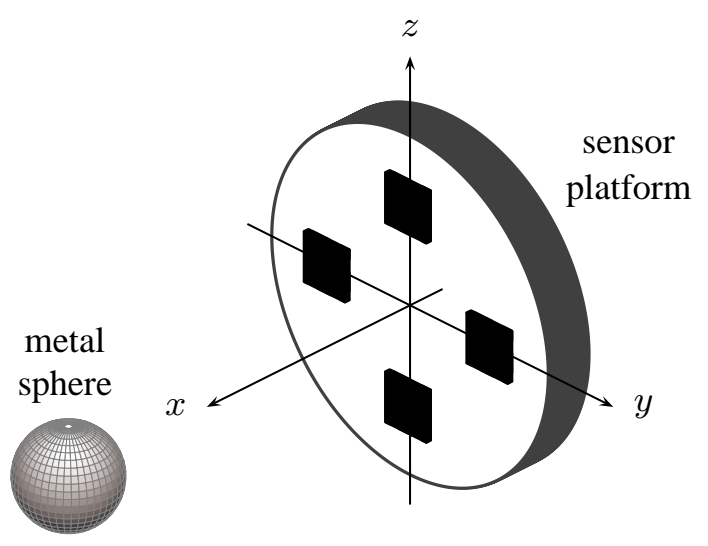

Figure 1. Concept of the toroidal holder with four modules in a symmetric arrangement and the internal coordinate system. A metal sphere serves as a measurement target.

symmetrical, perpendicular arrangement. The origin of the internal coordinate system is at the center point of the holder (sensor platform). Several modules are arranged around the milling machine tool to provide complete coverage of the three-dimensional surrounding space.

We designed a toroidal holder with integrated low-noise amplifiers (LNAs) which allows test scenarios with different antenna configurations involving up to five radar modules with variable radial distance, angle and positions in $x$ direction. Figure 2 shows the implementation with four radar modules and the corresponding integrated LNAs embedded in an open test environment. Some important properties of this additional system and the CW concept are its independence from the acting machine tool, robustness against environmental influences and reliability due to its simplicity.

Microwave sensorics is more suited for this application than cameras and optical sensors. This is due to the superior properties of microwaves with the above-mentioned problems with dirt and moisture in the operating space. Microwave sensorics is also more suited than acoustical and ultrasonic sensors. The speed of sound $\left(300 \mathrm{~mm} \mathrm{~ms}^{-1}\right)$ causes long signal delays compared to electromagnetic wave propagation.

Operation inside the enclosure of the machine and processing the diverse radar returns from there is one of the challenges for the signal processing. Multiple reflexions at the walls at arbitrary distances cause clutter effects and increase the noise level. Further challenges are the non-stationary environment, varying shapes and geometries of the milling tools as well as swarf from treatment in the operating zone.

In this first approach the system development concentrated on using CW radar only. This, on the one hand, is due to the robustness of the technology and the low cost arguments of the electronic hardware. On the other hand, the available space in any machine tool is limited. This only permits additional components of small size and low complexity. Fur-

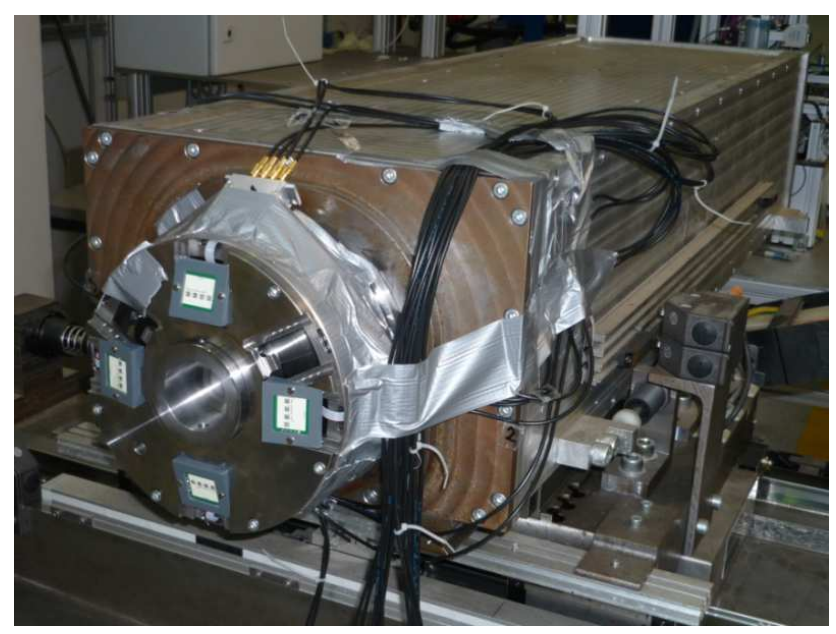

Figure 2. The implemented toroidal sensor platform equipped with four radars and integrated amplifiers embedded in an accessible, maneuverable test environment. Obtained signals are conducted via coaxial lines to the data storage and processing system.

thermore, the large operating temperature range and the rapid fluctuation of temperature must be dealt with by the equipment to meet the high demands on linearity of frequency ramps in frequency modulated CW radar (FMCW) or pulsed radar to provide proper distance information. Also the use of the $24 \mathrm{GHz}$ wideband technology with $5 \mathrm{GHz}$ bandwidth as in automotive SRR was ceased as of June 2013 (Strohm et al., 2005). The necessary bandwidth for a specific range resolution $\Delta r$ can be derived from $\Delta r=c_{0} /(2 \Delta f)$, where $c_{0}$ is the speed of light and $\Delta f$ is the absolute bandwidth. With the automotive SRR, a sufficient resolution of $3 \mathrm{~cm}$ is achievable if the full bandwidth of $5 \mathrm{GHz}$ is be used.

A second figure of merit is the accuracy $\delta r$ which is defined as (e.g., Skolnik, 1960; Barton, 1964),

$\delta r=\tau_{\mathrm{p}} \frac{c_{0}}{\sqrt{2 \mathrm{SNR}}}$,

with the pulse rise time $\tau_{\mathrm{p}}$ and the signal-to-noise ratio SNR. Just like the resolution, the accuracy depends on the bandwidth which determines $\tau_{\mathrm{p}} \approx 0.35 / \Delta f$. Both conditions, sufficient range resolution and accuracy, cannot be met in the 24 to $24.25 \mathrm{GHz}$ ISM-band.

\section{Designed multi-sensor Doppler radar network}

From the viewpoint of hardware one of the limiting factors in this application is the available installation space in the machine tool. A block diagram of a single sensor module and the signal processing components is given in Fig. 3 showing the RF front-end, which includes the $24 \mathrm{GHz}$ voltage controlled oscillator (VCO), a $90^{\circ}$ hybrid coupler, the transmitting (Tx) and receiving ( $\mathrm{Rx})$ 4-patch antenna array and the IQ mixing element. Due to the homodyne mixing process the 


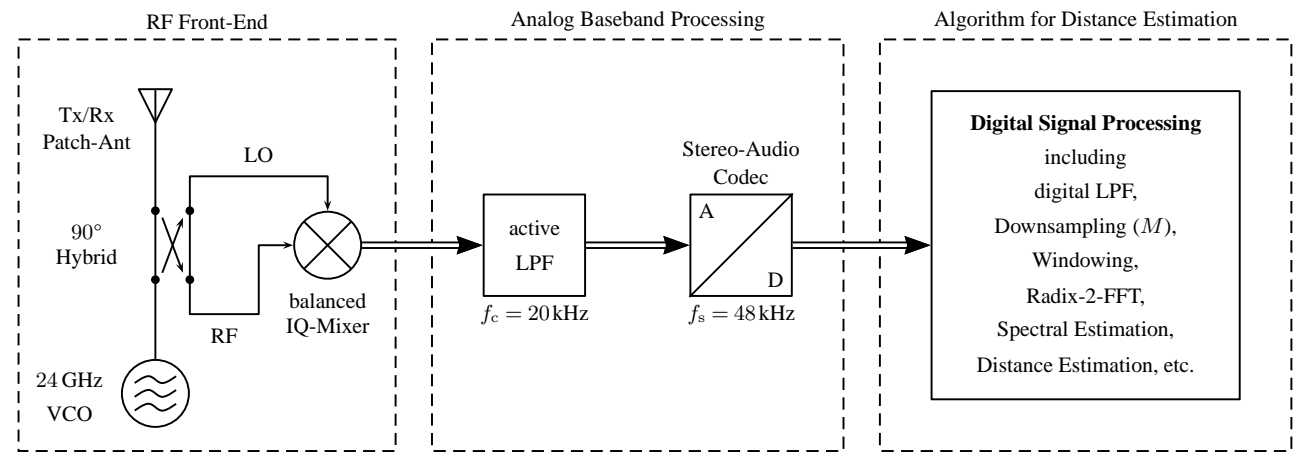

Figure 3. Block diagram of the radar system and signal processing components of a single module.

Table 1. Overview of several system parameters of the prototype.

\begin{tabular}{ll}
\hline system parameter & value \\
\hline supply voltage & $+5 \mathrm{~V}$ \\
power consumption & $\sim 4 \times 0.6 \mathrm{~W}$ \\
center frequency & $24.125 \mathrm{GHz}$ \\
antenna gain & $8.6 \mathrm{dBi}$ \\
transmit power & $+15 \mathrm{dBm}(\mathrm{EIRP})$ \\
half-power beamwidth & $80^{\circ}($ Azimuth $)$ \\
& $34^{\circ}($ Elevation $)$ \\
IF bandwidth & $3 \mathrm{~Hz}$ to $20 \mathrm{kHz}$ \\
IF gain & $40 \mathrm{~dB}$ \\
receiver sensitivity & $-80 \mathrm{dBm}$ \\
& $\left(B=20 \mathrm{kHz}, R_{\mathrm{IF}}=1 \mathrm{k} \Omega\right)$ \\
Doppler shift & $\left.160 \mathrm{~Hz} /(\mathrm{m} \mathrm{s})^{-1}\right)$ \\
sampling frequency & $48 \mathrm{kHz}$ \\
ADC resolution & $16 \mathrm{bit}$ \\
\hline
\end{tabular}

output of the module is the downconverted complex Doppler signal. A frequency gap of $30 \mathrm{MHz}$ between the carrier frequencies is introduced by tuning the VCOs to avoid crosstalk between the single modules of the radar network. Interfering down-conversion is then filtered out. The analog input stage consists of an active anti-aliasing low-pass filter (LPF) with a $3 \mathrm{~dB}$ cutoff frequency of $20 \mathrm{kHz}$, based on a low-noise operational amplifier. The input impedance is $1 \mathrm{k} \Omega$. This configuration allows a receiver sensitivity of $-80 \mathrm{dBm}$. A standard stereo-audio codec is used for converting the analog quadrature signal to a digital IQ data stream at a sampling frequency of $f_{\mathrm{s}}=48 \mathrm{kHz}$. Some system parameters of the radar module and the input stage are given in Table 1, compare RFbeam Microwave GmbH (2011).

As the radar system acts in severe environmental conditions with fluid, drizzle, heat etc., hermetically sealed modules are necessary. The enclosures of the radar front-end in Fig. 2 are made from polyethylene and are closed and sealed for the final application. Additionally, they increase the flexibility for positioning and tilting of the modules. Coverage simulations based on deterministic ray-tracing showed that $15^{\circ}$ tilting of the modules gives best coverage of the surrounding space around the tool (Azodi et al., 2013).

The input stage is built into a metal shielding enclosure. It is supposed to protect the circuit not only against electromagnetic interference but also against the harsh environmental conditions in machine tools. Experiments showed that interference from power supplies, switching electrical actuators and mechanical vibrations are there to effectively increase the noise level. The high-frequency interference can be suppressed after data acquisition by digital filtering as described in the next section. Low frequency components in the subkilohertz range remains as superimposed interfering signals.

\section{Implemented digital signal processing stage}

As the radar modules are based upon a homodyne architecture, the IQ mixer output appears in baseband centered around DC. The analog bandwidth of the active LPF is from $3 \mathrm{~Hz}$ to $20 \mathrm{kHz}$. This covers the needed range of relative velocities especially for slowly moving targets.

After digital conversion the signal is buffered and the DC bias is removed. Next a decimation stage follows. It consists of a digital LPF and subsequent downsampling by factor of $M$. Figure 4 illustrates the preprocessing steps between ADC and spectral frequency estimation. The digital LPF is a 4th order Chebyshev type I filter with $0.25 \mathrm{~dB}$ ripple and $f_{\mathrm{c}}=160 \mathrm{~Hz}$ cutoff frequency. This cutoff equates to the Doppler shift due to the highest expected relative velocity of $1 \mathrm{~m} \mathrm{~s}^{-1}$. The filter process reduces the noise contribution, hence the sensitivity level is decreased down to $-101 \mathrm{dBm}$.

Each observation window has length $N T$ seconds and is weighted with a window function after decimation. $T=1 / f_{\mathrm{s}}$ is the sampling period and $N$ is the number of sampling points. The decimation process does not change the observation period. The new sampling period is $T_{\mathrm{M}}=M T=M / f_{\mathrm{s}}$ and the number of sampling points is $N / M$. A Kaiser window with $80 \mathrm{~dB}$ sidelobe suppression has proven sufficient selectivity and low spectral leakage. The Kaiser window shape parameter value was chosen as $\alpha=3.422$. 


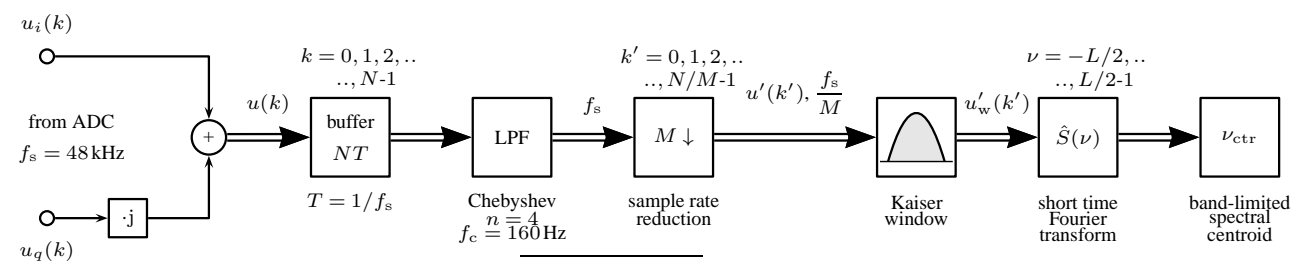

Figure 4. Preprocessing of complex signal from one single path.

Frequency resolution is determined by the duration of observation. The number of sampling points for each segment are kept to $N \sim 2^{n}$ for utilization of the efficient Radix-2FFT algorithm. To extract Doppler shift and relative velocity, the short time Fourier spectrum is derived from each data segment by the estimator

$\hat{S}(v)=\sum_{k^{\prime}=0}^{L-1} u_{\mathrm{w}}^{\prime}\left(k^{\prime}\right) \exp \left(-j 2 \pi v k^{\prime} / L\right)$,

where $k^{\prime}$ is an integer and $u_{\mathrm{w}}^{\prime}\left(k^{\prime}\right)$ is the preprocessed signal. The Fourier spectrum is calculated at discrete points $v$, where $v=-L / 2, \ldots, L / 2-1$ and $L=N / M$.

Frequency estimation is based on computation of the bandwidth limited spectral centroid. The bandwidth for calculation is limited to a window which is identical to the LPF passband, excluding a small guard interval around DC. Exploiting the sign of the received Doppler shift provided by the IQ mixer architecture the integration limits can be set to the frequency indices $v_{\min }<v<v_{\max }$ where all indices are positive for positive Doppler shifts and negative for negative Doppler shifts. The spectral centroid is then calculated only in the frequency interval of interest by

$v_{\mathrm{ctr}}=\frac{\sum_{\nu=\nu_{\min }}^{\nu_{\max }} v|\hat{S}(v)|^{2}}{\sum_{\nu=v_{\min }}^{\nu_{\max }}|\hat{S}(v)|^{2}}$.

Limiting the summation in Eq. (3) reduces noise to a minimum and prevents erroneous biased estimation. In case of multi-target scenarios different methods must be utilized regarding more than one spectral maximum. But this case is not discussed in this paper since first investigations and performance tests assume that there is a single dominant target.

From $v_{\text {ctr }}$ the value of $\hat{f}_{\mathrm{d}}=v_{\mathrm{ctr}} f_{\mathrm{s}} /(L M)$ is calculated. An estimate for the relative velocity can be obtained with the well-known equation $\hat{v}_{\text {rel }}=\hat{f}_{\mathrm{d}} \lambda / 2$, where $\lambda=c_{0} / f_{\mathrm{c}}$ is the wavelength of the corresponding carrier frequency $f_{\mathrm{c}}$.

Figure 5 shows a typical spectrogram acquired from the observation of the space in front of a sensor with one single dominant target. This example is based on real measurements performed with the prototype shown in Fig. 2. In this case the sensor platform is centrally arranged to the sphere and first approaches to it and afterwards departs from it. The spectrogram in Fig. 5 shows a distinct main frequency component.

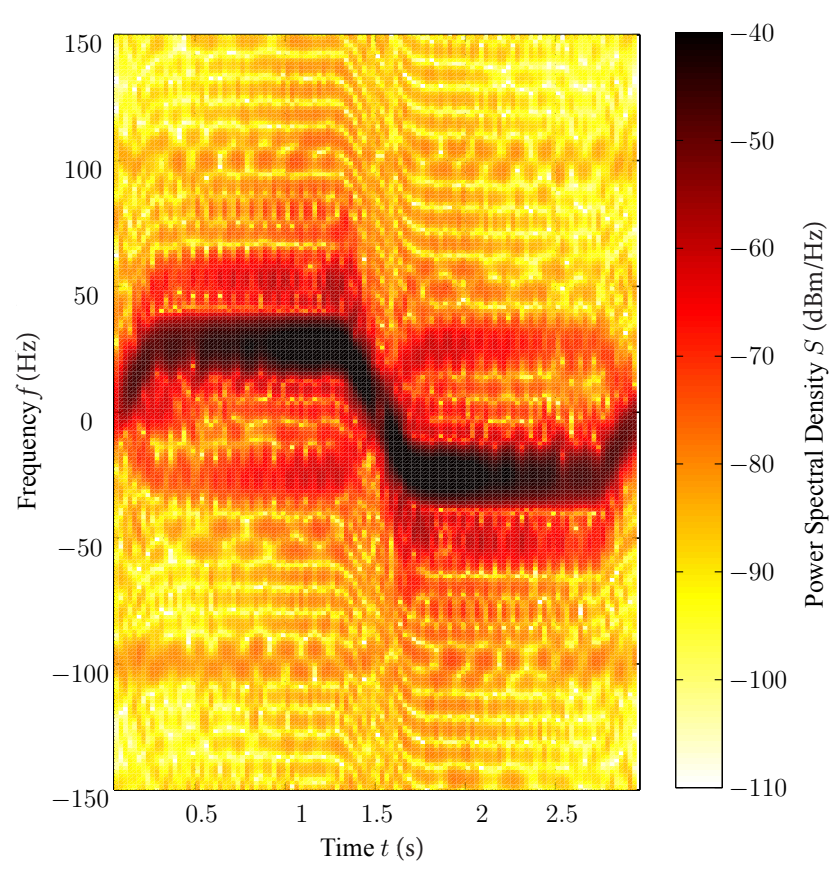

Figure 5. Doppler spectrogram for a closing and receding movement of the sensor platform. A metallic sphere serves as reflecting object.

Short phases of acceleration and deceleration at the beginning and the end and a turning point can be identified.

A critical point for unambiguous detection and tracking of objects are spurious frequency components that are observable in Fig. 5. These artifacts arise from IQ imbalance. The origins of this analog signal distortion are fabrication tolerances of the Schottky diodes and the transmission lines in the mixer stage. Shifts or distortions of the diode characteristic on the one hand and slightly unequal power division or phase deviation from ideal dividers on the other hand are the consequences. This causes variations between the output signals of the two mixing units which results in degraded signal quality. Like clutter, spurious peaks potentially increase the false alarm rate considerably. Especially, if solids with fluctuating radar cross section (RCS) have to be detected, e.g. cuboids or cylinders, image components will influence the algorithm performance of the decision unit when this special problem remains unconsidered. This issue is investigated in detail in future work. 


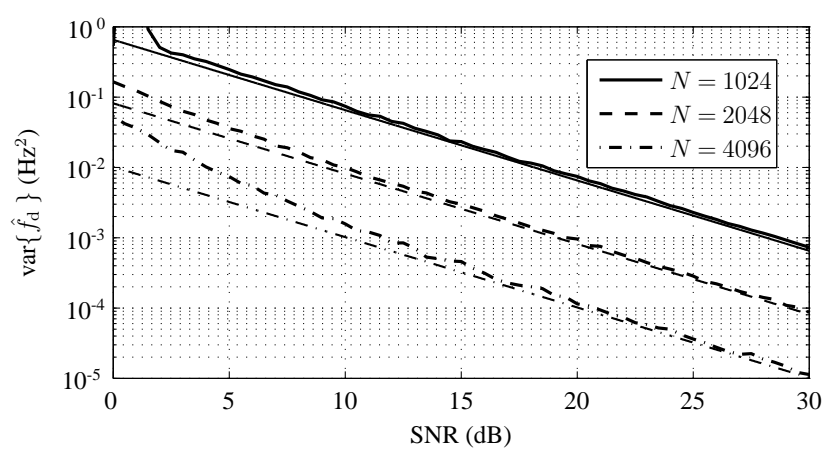

Figure 6. Frequency estimation simulation results for a single complex sinusoid with unknown amplitude and phase and the theoretical bounds for estimation variances. $f_{\mathrm{s}}=48 \mathrm{kHz}, f_{\mathrm{d}}=25.6 \mathrm{~Hz}$.

\section{Single target scenario}

In a single target scenario with one dominant scatterer at position $\boldsymbol{r}_{0}=\left(x_{0}, y_{0},\right)^{\mathrm{T}}$ the observed discrete-time Doppler shift signal detected by the $i$ th sensor is modeled by

$u^{i}(k)=A^{i} \exp \left(\mathrm{j}\left(\Omega_{\mathrm{d}}^{i} k+\varphi^{i}\right)\right)+w^{i}(k)$,

where $\Omega_{\mathrm{d}}=\omega_{\mathrm{d}} T$ is the normalized Doppler frequency and $w$ is band-limited measurement and processing noise. Measurements reveal that $w$ is Gaussian white noise with the normal distribution

$p(\boldsymbol{w}) \sim \mathcal{N}(0, \mathbf{C})$,

where $\mathbf{C}$ is the noise covariance matrix.

In this application the parameters $A, \omega_{\mathrm{d}}$ and $\varphi$ are all unknown. In the following the index $i$ is omitted. The CramerRao-Bound (CRB) is a lower bound on the minimum achievable variances depending on the signal properties. In (Rife and Boorstyn, 1974) results for a discrete-time single complex sinusoid can be found. The CRB on the variance of the Doppler frequency estimate $\hat{f}_{\mathrm{d}}=\hat{\omega}_{\mathrm{d}} /(2 \pi)$ is given by

$\operatorname{var}\left\{\hat{f}_{\mathrm{d}}\right\}=\frac{12 \sigma_{N}^{2}}{(2 \pi)^{2} A^{2} T^{2} N\left(N^{2}-1\right)}$,

where $\sigma_{N}^{2}$ is the variance of the noise. The variance of $\hat{f}_{\mathrm{d}}$ depends on the $\mathrm{SNR}=A^{2} / \sigma_{N}^{2}$ and the observation time $N T$ which is determined by a trade-off between accuracy and agility of signal processing algorithm. Simulation results for a noisy complex sinusoid with varying SNR and various numbers of sampling points $N$ are illustrated in Fig. 6, together with the theoretical lower bounds given by Eq. (6).

For low SNR the frequency estimation is distorted and the variance rapidly increases. In Tab. 2 the values for three standard deviations $3 \sigma$ of the estimated mean frequency are given. This range includes $99.7 \%$ of all estimates.

The obtained Doppler frequency shift depends on the sensor-target geometry via the inner product of the distance
Table 2. Simulation results for the standard deviation: $f_{\mathrm{s}}=48 \mathrm{kHz}$, $f_{\mathrm{d}}=25.6 \mathrm{~Hz}, \mathrm{SNR}=10 \mathrm{~dB}$.

\begin{tabular}{cccc}
\hline$N$ & 1024 & 2048 & 4096 \\
\hline $3 \sigma / \mathrm{Hz}$ & 0.8161 & 0.2999 & 0.1190 \\
$3 \sigma_{\mathrm{crb}} / \mathrm{Hz}$ & 0.7662 & 0.2709 & 0.0958 \\
\hline
\end{tabular}

vector $\left(\boldsymbol{r}_{0}-\boldsymbol{s}\right)$ and the velocity vector $\boldsymbol{v}=\left(v_{x}, v_{y}, v_{z}\right)^{\mathrm{T}}$. The equation for the general three-dimensional case with sensor at position $\left(s_{x}, s_{y}, s_{z}\right)^{\mathrm{T}}$ is

$f_{\mathrm{d}}=\frac{2}{\lambda} \frac{\left(\boldsymbol{r}_{0}-\boldsymbol{s}\right)^{\mathrm{T}} \boldsymbol{v}}{\left\|\boldsymbol{r}_{0}-\boldsymbol{s}\right\|}$,

where $\|\cdot\|$ represents the standard Euclidean vector norm.

\subsection{Data model}

In this application a special kind of observation scenario arises. Several sensors are closely spaced on a maneuverable platform monitoring the space in front with one dominant scatterer. Figure 7 illustrates the scenario with sensor platform and object inside the closed machine. The platform typically moves with constant velocity on straight-lined trajectories.

Compilation of Eq. (7) for a radar network with several distributed sensors results in a system of nonlinear equations

$f_{\mathrm{d}}=\mathbf{A}(\xi)+\boldsymbol{w}$.

with the observation matrix $\mathbf{A}(\boldsymbol{\xi})$ which contains the the model of Eq. (7) and the parameter vector $\xi=$ $\left(x, y, z, v_{x}, v_{y}, v_{z}\right)^{\mathrm{T}}$ for the general three-dimensional case. The number of unknowns is reduced to four as the twodimensional case is considered. To find the optimal $\xi^{*}$ the constrained minimization problem (e.g., Bjoerk, 1996),

$\underset{\xi}{\operatorname{minimize}} F(\xi)$

such that $g(\xi) \leq b_{\ell}, \quad \ell=1, \ldots, p$

has to be solved, where $F(\boldsymbol{\xi})$ is the cost function and $\boldsymbol{g}(\boldsymbol{\xi})$ contains linear and nonlinear constraints for maximum distances between sensor and object and maximum velocities in all directions. To solve this nonlinear minimization problem and to find a solution for position $\boldsymbol{r}_{0}$ and velocity of the platform $\boldsymbol{v}$, Newton-type or Gauss-Newton method is applied. A fundamental problem is to find the global minimum. For cost functions and their Jacobian with several minima, the accuracy of the results strongly depend on the initial guess. More advanced methods are necessary which find the global minimum, e.g. grid search methods. These account for higher computing capacity at the beginning of a new trajectory. Then, the previously found solutions can be used recursively. The problem is investigated in detail in future works. 


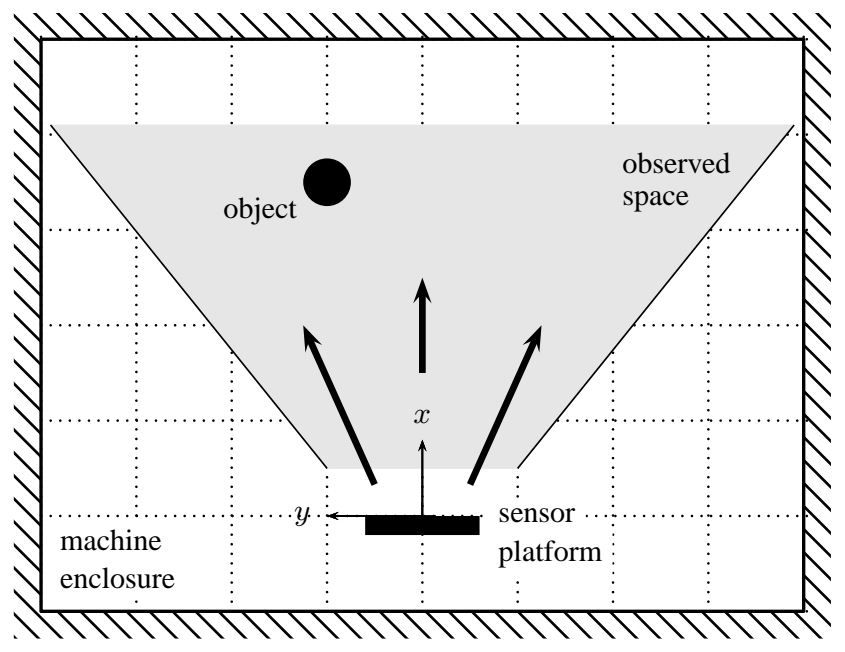

Figure 7. Illustration of a typical scenario of the application: One dominant scatterer at fixed position and the maneuverable sensor platform with radar modules. Arrows point out the typical rectilinear motion during manual operation.

In the next section measurement data of a one-dimensional linear motion are analyzed to show the accuracy of the frequency estimation. The second example investigates a twodimensional scenario. A nonlinear solver is used to obtain position estimates from synthetic and measurement data.

\subsection{Measurement results for linear motion}

This section provides the results of the algorithm applied to a set of measurements. The measurements are done with the in-house developed prototype depicted in Fig. 2. They clarify quality and accuracy of the algorithm for frequency estimation. The received signal streams are stored and available for off-line post processing. To obtain sufficient frequency resolution the processed block size is set to $N=4096$ data points. This results in a resolution of $1 /(N T) \approx 11.7 \mathrm{~Hz}$ during the post processing. The decimation process has no influence on the value of $N T$. Variable update rates are achieved by overlapped data segments. Measurement and reference data for position and speed of the sensor platform with the same update rate of $4 \mathrm{~ms}$ are used for performance tests with the prototype. Reference data available from the machine tool control unit provides exact position and velocity data.

Results of the following scenario are presented: Comparable to Figs. 1 and 2, the sensor platform is equipped with four sensors. The movement of the sensor platform is bidirectionally (i.e. back and forth) on an axial path. A metal sphere with a diameter $d=15 \mathrm{~cm}$ serves as scattering object. Its position is fixed and aligned to the center of the platform. As the circumference of the sphere is much larger than the wavelength, the RCS of the sphere is in the optical region and equal the cross-section area $d^{2} \pi / 4$ of the sphere.

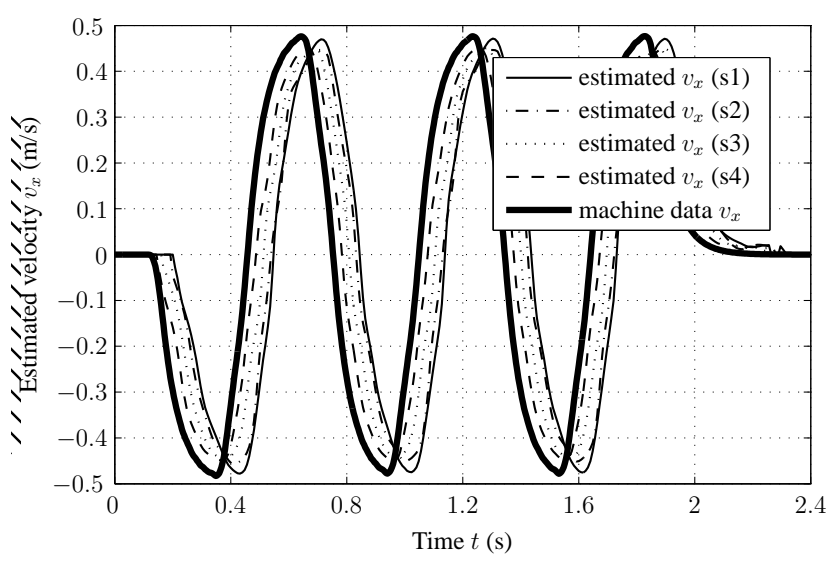

Figure 8. Estimated velocity from Doppler shift measurements for an axial movement of the sensor platform.

Referred to the coordinate system in Fig. 1 the velocitiy components $v_{y}$ and $v_{z}$ of the platform are both equal to zero and the $y$ and $z$ coordinates remain constant. Figure 8 shows the obtained velocity from the internal machine's control unit (solid graph) and the estimated velocity $v_{x}$ (dashed and dotted graphs) from the data of each of the four sensors. The small time shifts between the various sensor streams come from the data acquisition procedure. The exact value is known and can be compensated. At the beginning a receding movement is identified (negative sign) with continuously increasing velocity up to a deceleration phase around 0.35 to $0.45 \mathrm{~s}$. Then, the turning point is reached and the movement with positive acceleration and velocity follows (positive sign). The movement pattern is repeated three times. Against the background of a dynamic scenario with everchanging velocity, the estimates are in very good agreement with the true velocity in Fig. 8. In this scenario velocities of $0.47 \mathrm{~m} \mathrm{~s}^{-1}$ and accelerations up to $6.5 \mathrm{~m} \mathrm{~s}^{-2}$ are reached.

Minor discrepancy can be identified at the turning points where the velocities and change in position becomes small. This is due to the limited frequency resolution. Frequencies lower than $11.7 \mathrm{~Hz}$ (corresponding to $\sim 73.1 \mathrm{~mm} \mathrm{~s}^{-1}$ in this system) are actually mapped to zero due to lack of accuracy of the estimate. Improvements are achieved by using adaptive parallel analyzing of longer sequences and subsequent adjustment of the position.

\subsection{Results for diagonal motion}

As a further scenario a two-dimensional motion is investigated. Figure 9 shows the results for a diagonal approaching motion where $v_{x}$ and $v_{y}$ are nonzero. Using the coordinate system of Fig. 1, the starting position of the object (sphere, $d=15 \mathrm{~cm})$ is at $(0.5 \mathrm{~m}, 0.5 \mathrm{~m}, 0)^{\mathrm{T}}$ and the constant velocity of the sensor platform is $\left(-0.707 \mathrm{~m} \mathrm{~s}^{-1},-0.707 \mathrm{~m} \mathrm{~s}^{-1}, 0\right)^{\mathrm{T}}$. The position of the object is represented by circles. The results for synthetic data $(+)$ and for real measurement data 


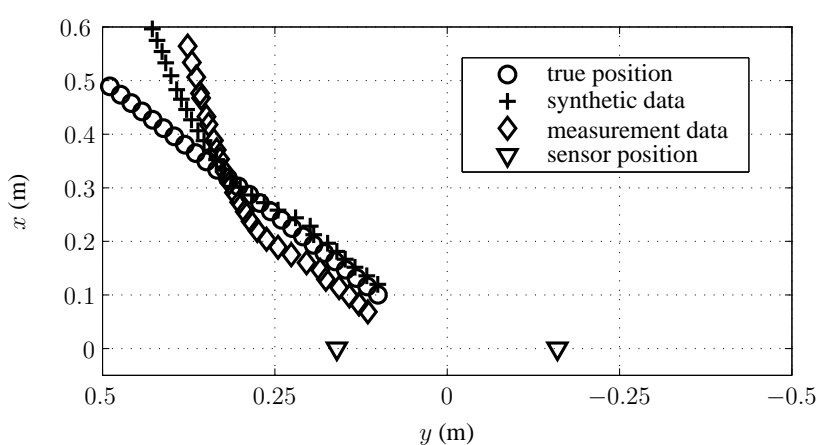

Figure 9. Target localization close to the sensor platform. The initial guess of the parameter vector for the iterative nonlinear solver is $\left(0.91 \mathrm{~m}, 0.53 \mathrm{~m}, 0,-0.66 \mathrm{~m} \mathrm{~s}^{-1},-0.33 \mathrm{~m} \mathrm{~s}^{-1}, 0\right)^{\mathrm{T}}$.

$(\diamond)$ are very similar and close to the real position of the object.

The algorithm is based on the approach of Chan and Jardine (1990) which uses a special cost function involving the time derivative of the Doppler shift $\dot{f}_{\mathrm{d}}$ (rate of change). For further details the reader is referred to the original paper.

In this example the absolute errors between real and estimated position are lower than $4 \mathrm{~cm}$, what is already sufficient for collision warning in machine tools. Further investigations are in progress to improve reliability and flexibility of the algorithm to make it applicable for arbitrary linear motions.

\section{Conclusions}

This paper presented an implementation of a prototype system that aims at a new application of $24 \mathrm{GHz} \mathrm{CW}$ radar technology in machine tools of collision detection and avoidance. Important aspects for the mechanical design, the RF hardware, the components for the baseband processing and the elements of the developed algorithm for velocity estimation have been described. This first approach treats singletarget scenarios in a two-dimensional space. For validation the case of linear motion has been investigated. Results from conducted measurements reveal very good agreement of velocity estimates with the reference data provided by the machine's control unit. The application of a nonlinear solver for position estimation in a two-dimensional motion scenario showed acceptably low position estimation errors. This prototype system serves as the basis for further investigations and developments towards a multi-sensor radar system for fast collision detection in machine tools. Due to the rather weak sensitivity of the used modules and the coarse Doppler resolution due to short observation windows the effect of multiple targets and multiple reflections has not been considered. It is expected that this simple approach is suitable for the majority of collision scenarios. However, specification of the actual false alarm and missed detection rates requires further studies. Further topics for future work are ex- tension to three dimensions, the question of the minimum necessary number of sensors for uniqueness of the solution, and their optimum positioning around the tool. Although the observation time is very short in this application the effect of cross section fluctuations also needs further investigations.

Acknowledgements. The authors would like to thank M. Berger and D. Korff from the Institute of Production Management, Technology and Machine Tools (PTW) of the Technische Universität Darmstadt for their help and support during the execution of the measurements.

This work is funded by the Bavarian Ministry of Economic Affairs and Media, Energy and Technology within the economic development scheme "Mikrosystemtechnik Bayern" under grant BAY158/002.

Edited by: U. van Rienen

Reviewed by: two anonymous referees

\section{References}

Abele, E., Brecher, C., Gsell, S. C., Hassis, A., and Korff, D.: Steps towards a protection system for machine tool main spindles against crash-caused damages, Springer J. Prod. Eng., 6.6, 631-642, doi:10.1007/s11740-012-0422-6, 2012.

Azodi, H., Siart, U., and Eibert, T. F.: A fast three-dimensional deterministic ray tracing coverage simulator for a $24 \mathrm{GHz}$ anticollision radar, Adv. Radio Sci., 11, 55-60, doi:10.5194/ars-1155-2013, 2013.

Barton, D. K.: Radar System Analysis, 1. Ed., Prentice Hall, 1964.

Björck, Å.: Numerical Methods for Least Squares Problems, 1. Ed., Society for Industrial and Applied Mathematics (SIAM), doi:10.1137/1.9781611971484, 1996.

Chan, Y.-T. and Jardine, F. L.:, Target localization and tracking from Doppler-shift measurements, IEEE J. Oceanic Eng., 15, 251257, doi:10.1109/48.107154, 1990.

RFbeam Microwave GmbH: K-LC2 Radar Transceiver - Datasheet, available at: http://www.rfbeam.ch (last access: 12 July 2013), 2011.

Rife, D. and Boorstyn, R. R.: Single tone parameter estimation from discrete-time observations, IEEE Trans. Inf. Theory, 20, 591598, doi:10.1109/TIT.1974.1055282, 1974.

Skolnik, M. I.: Theoretical accuracy of radar measurements, IRE Trans. Aeronaut. Navig. Electron., ANE-7.4, 123-129, doi:10.1109/TANE3.1960.4201757, 1960.

Strohm, K. M., Bloecher, H.-L., Schneider, R., and Wenger, J., Development of future short range radar technology, European Radar Conference 2005, 165-168, doi:10.1109/EURAD.2005.1605591, 2005. 